\title{
Levantamento visual contínuo: análise da rodovia TO-255, trecho de Porto Nacional a Monte do Carmo
}

O presente projeto propôs uma avaliação das condições da superfície do pavimento flexível da rodovia TO-255, trecho de Porto Nacional a Monte do Carmo - TO contabilizando 39,1 quilômetros. Para realizar a pesquisa aplicou-se o método de avaliação superficial de pavimento flexível denominado Levantamento Visual Contínuo - LVC, desenvolvido pelo DNIT e regido pela norma 008/2003 - PRO na qual informa as condições necessárias para a realização do método, que ocorre a partir de visitas in loco para identificar as principais patologias do trecho. 0 método baseia-se na verificação do estado geral da superfície asfáltica e das manifestações patológicas nela presentes, resultando-se em 03 (três) índices que são: ICPF (Índice de Condição de Pavimentos Flexíveis), IGGE (Índice de Gravidade Global Expedito) e o IES (Índice do Estado de Superfície do Pavimento). Foram analisados quarenta subtrechos com extensão de um quilômetro cada, e através dessa pesquisa foi possível classificar o estado de conservação do pavimento atual como ótimo, bom, regular, ruim e péssimo, bem como a frequência das patologias identificadas no seguimento. Á partir dessa análise é evidenciada a importância de planejamentos que envolvam medidas de conservação e prevenção sobre o pavimento, evitando o agravamento das condições da via, possibilitando assim um gerenciamento consciente e eficaz.

Palavras-chave: Patologias; Pavimentos Flexíveis; Levantamento Visual Contínuo; Restaurações Asfálticas.

\section{Continuous visual survey: analysis of the TO-255 highway, from Porto Nacional of Monte do Carmo}

\begin{abstract}
The present project proposed an evaluation of the surface conditions of the flexible pavement of the TO-255 highway, from Porto Nacional to Monte do Carmo TO, accounting for 39.1 kilometers. In order to carry out the research, the method of surface evaluation of flexible pavement called Continuous Visual Survey - LVC, developed by DNIT and ruled by the standard 008/2003 - PRO was applied, in which it informs the necessary conditions for the accomplishment of the method, that occurs from of visits in loco to identify the main pathologies of the stretch. The method is based on the verification of the general state of the asphalt surface and the pathological manifestations present in it, resulting in 03 (three) indices that are: ICPF (Flexible Pavement Condition Index), IGGE (Global Expedited Gravity Index) and the IES (Index of Pavement Surface State). Forty surcharges with an extension of one kilometer each were analyzed, and through this research it was possible to classify the state of conservation of the current pavement as Great, Good, Fair, Poor and Poor, as well as the frequency of pathologies identified in the follow - up. Based on this analysis, the importance of planning that involves conservation and prevention measures on the pavement is evidenced, avoiding the worsening of the road conditions, thus allowing a conscious and effective management.
\end{abstract}

Keywords: Pathologies; Flexible Flooring; Continuous Visual Survey; Asphalt Restorations.

Topic: Engenharia Civil

Reviewed anonymously in the process of blind peer.

Danyelle Crystinne Mecenas Ferreira dos Santos Instituto Tocantinense Presidente Antônio Carlos, Brasil

http://lattes.cnpq.br/3132605237775524

dane.moreiraesantosadvogados@gmail.com

\section{Flávio Vieira da Silva Júnior}

Instituto Tocantinense Presidente Antônio Carlos, Brasil

http://lattes.cnpq.br/2211436659738090

flaviovisiju@gmail.com

d

DOI: $10.6008 / C B P C 2318-3055.2018 .001 .0002$
Received: $\mathbf{1 1 / 0 1 / 2 0 1 8}$

Approved: 20/03/2018
Referencing this:

SANTOS, D. C. M. F.; SILVA JÚNIOR, F. V.. Levantamento visual contínuo: análise da rodovia TO-255, trecho de Porto Nacional a Monte do Carmo. Engineering Sciences, v.6, n.1, p.10-20, 2018. DOI: http://doi.org/10.6008/CBPC2318-3055.2018.001.0002 


\section{INTRODUÇÃO}

O Estado do Tocantins possui atualmente $2.117,2$ quilômetros de rodovias federais implantadas e mais de 6000 quilômetros de rodovias estaduais pavimentadas, muito além da realidade da época de sua criação quando possuía apenas 228 quilômetros de rodovias estaduais pavimentadas (CNT, 2017).

O estado passou desde sua criação por uma enorme expansão econômica, chegando a aumentar quase três vezes sua renda per capita no período de uma década. A não previsão no projeto devido este crescimento repentino afetou diretamente o modal rodoviário. Com o aumento da solicitação de cargas favoreceu o surgimento de falhas, tornando o pavimento defeituoso em razão das patologias ocorrentes por desgaste da superfície, prejudicando o bom funcionamento da via (BORGES et al., 2013).

A Confederação Nacional dos Transportes - CNT (2017) em uma análise do pavimento de âmbito nacional, utilizando dentre os métodos a ferramenta de levantamento visual continuo do DNIT, observou, que dos 1.569 quilômetros de rodovias estaduais analisados no Tocantins, classifica como regular $87,5 \%$ das vias estaduais, de acordo com a tabela 1.

Tabela 1: Classificação do pavimento: por tipo de jurisdição.

\begin{tabular}{|c|c|c|c|c|}
\hline \multirow{2}{*}{ CLASSIFICAÇÃO DO PAVIMENTO } & \multicolumn{2}{|c|}{ EXTENSÃO FEDERAL } & \multicolumn{2}{|c|}{ EXTENSÃO ESTADUAL } \\
\hline & $\mathrm{km}$ & $\%$ & $\mathrm{~km}$ & $\%$ \\
\hline Ótimo & 876 & 52,4 & 0 & 0 \\
\hline Bom & 139 & 8,3 & 4 & 0,3 \\
\hline Regular & 586 & 35 & 1.374 & 87,5 \\
\hline Ruim & 52 & 3,1 & 138 & 8,8 \\
\hline Péssimo & 20 & 1,2 & 53 & 3,4 \\
\hline TOTAL & 1.673 & 100 & 1.569 & 100 \\
\hline
\end{tabular}

Fonte: CNT (2017).

É notório que as más condições encontradas se devem à carência de conservação e manutenção, fato que provoca riscos aos usuários, além de aumentos nos custos da economia regional. Este trabalho teve como proposta analisar as patologias do trecho da TO-255 que liga Porto Nacional - TO a Monte do Carmo TO, utilizando o método do Levantamento Visual Contínuo - Procedimento DNIT 008/2003 (DNIT, 2003).

Realizou-se ainda o levantamento das principais patologias encontradas no seguimento estudado, identificando as possíveis origens da deterioração do pavimento e propondo soluções técnicas adequadas para as patologias encontradas no trecho analisado.

\section{METODOLOGIA}

A pesquisa ocorreu no trecho da TO-255 que liga Porto Nacional - TO a Monte do Carmo - TO, descrita na Figura 1, levando em consideração além da importância econômica para região, o fluxo de veículos. O trecho em estudo é uma rodovia estadual com uma extensão de 39,1 km, no qual possui uma pista simples de mão dupla. Localizado na região central do estado do Tocantins e está situado a $69 \mathrm{~km}$ da capital do estado, Palmas - TO.

O método escolhido para realização do estudo foi o Levantamento Visual Contínuo (LVC) normatizado pelo DNIT 008/2003 - PRO, no qual tem como objetivo realizar a avaliação de pavimentos 
flexíveis e semirrígidos partir da definição do Índice de Condição dos Pavimentos Flexíveis (ICPF) que está descrito no item 2.2.

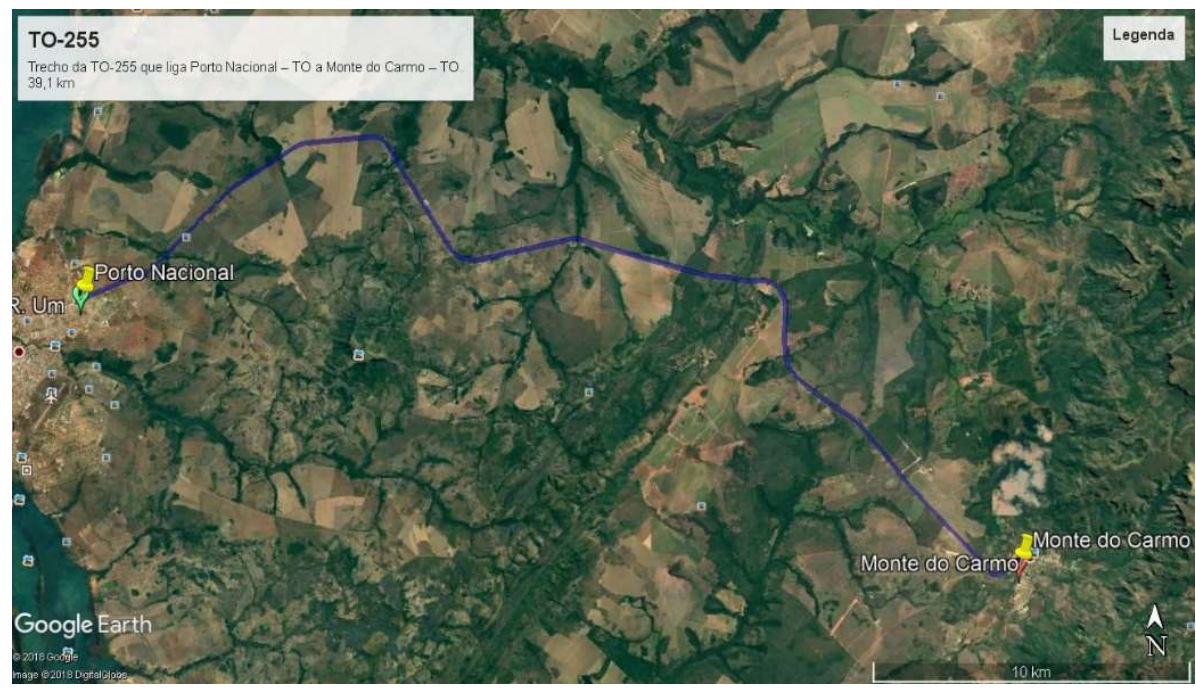

Figura 1 - Demarcação do trecho de Porto Nacional a Monte do Carmo.

Com isso, realizou-se um levantamento de campo das patologias presentes no pavimento do trecho escolhido, seguido de uma análise dos resultados obtidos. Foram identificadas as possíveis origens, e por fim, apresentadas soluções técnicas para as falhas encontradas.

\section{Condições do Levantamento Visual Contínuo}

Foi utilizado um carro equipado com velocímetro/odômetro calibrado a partir de um GPS, evitando a realização do procedimento em dias chuvosos, com neblina, e com pouca luz natural. O veículo estava manipulado a uma velocidade média aproximada de $40 \mathrm{~km} / \mathrm{h}$, percorreu pela via em um único sentido e levou-se em consideração simultaneamente as duas faixas de tráfego. O processo de levantamento visual contínuo compreende o preenchimento de alguns formulários. Maiores informações sobre a extensão dos segmentos e o preenchimento dos formulários são descritas pelo DNIT 008/2003 - PRO.

\section{ICPF: Índice de Condição dos Pavimentos Flexíveis}

O cálculo é originado mediante média dos índices inclusos no Formulário do Levantamento. Este índice é feito por meio da conceituação do observador em relação à precisão de um aprimoramento na camada superficial do pavimento. Para cada subtrecho avaliado, os resultados dos cálculos do ICPF são anotados no Quadro de Resumo disponibilizado pelo DNIT 008/2003 - PRO.

\section{IGGE: Índice De Gravidade Global Expedido}

Seguindo o mesmo método da norma DNIT 006/2003 PRO, o cálculo do IGGE é obtido através do desenvolvimento da fórmula a seguir, no qual deve ser desenvolvida com o auxílio dos dados do Formulário de cálculo do IGGE (Quadro 1), analisado pelas condições descritas no item 2.1. Os resultados foram preenchidos no Quadro de Resumo. 


$$
I G G E=(P t \times F t)+(P o a p \times F o a p)+(P p r \times F p r)
$$

Onde:

- Ft = Frequência do conjunto das trincas; - $P t=$ Peso do conjunto das trincas;

- Foap = Frequência do conjunto de deformações;

- Poap = Peso do conjunto de deformações;

- $F p r=$ Frequência (quantidade por $\mathrm{km}$ ) do conjunto de panelas e remendos; - Ppr $=$ Peso do conjunto de panelas e remendos.

Os pesos para o cálculo do IGGE em função da frequência e da gravidade das situações das falhas anotadas no formulário para o Levantamento Visual Contínuo, são disponibilizados nas Tabelas 2 e 3 abaixo.

Tabela 2: Determinação do índice de gravidade.

\begin{tabular}{ccc}
\hline & Panelas (P) e Remendos (R). & GRAVIDADE \\
\hline FREQUÊNCIA & Fpr Quantidade/Km & 3 \\
\hline A - ALTA & $\geq 5$ & 2 \\
\hline M - MÉDIA & $2-5$ & 1 \\
\hline B - BAIXA & $\leq 2$ & \\
\hline FREQUÊNCIA & Ft e Foap (\%) & GRAVIDADE \\
\hline A - ALTA & $\geq 50$ & 3 \\
\hline M - MÉDIA & $50-10$ & 2 \\
\hline B - BAIXA & $\leq 10$ & 1 \\
\hline
\end{tabular}

Fonte: DNIT (2003).

Tabela 3: Pesos para cálculo.

\begin{tabular}{cccc}
\hline GRAVIDADE & Pt & Poap & Ppr \\
\hline 3 & 0,65 & 1 & 1 \\
\hline 2 & 0,45 & 0,70 & 0,80 \\
\hline 1 & 0,30 & 0,60 & 0,70 \\
\hline
\end{tabular}

Fonte: DNIT (2003).

\section{IES: Índice do Estado da Superfície do Pavimento}

Os valores do IES são compreendidos de 0 a 10, sendo qualificado em função do IGGE e do ICPF calculados, sendo uma síntese destes dois índices. Os resultados obtidos de cada subtrecho analisado, são anotados no Quadro de resumo disponibilizado pela norma DNIT 008/2003 - PRO. Os valores do IES juntamente com o Código e o Conceito atribuídos ao estado da superfície do pavimento são determinados com os valores que constam na Tabela 4.

Tabela 4: IES - Índice do Estado da Superfície do pavimento.

\begin{tabular}{cccc}
\hline DESCRIÇÃO & IES & CÓDIGO & CONCEITO \\
\hline IGGE $\leq 20$ e ICPF $>3,5$ & 0 & A & ÓTIMO \\
\hline IGGE $\leq 20$ e ICPF $\leq 3,5$ & 1 & \multirow{2}{*}{ BOM } \\
\hline $20 \leq \mathrm{IGGE} \leq 40$ e ICPF $>3,5$ & 2 & \multirow{2}{*}{ REGULAR } \\
\hline $20 \leq \mathrm{IGGE} \leq 40$ e ICPF $\leq 3,5$ & 3 & \multirow{2}{*}{$\mathrm{C}$} & $\mathrm{RUIM}$ \\
\hline $40 \leq \mathrm{IGGE} \leq 60$ e ICPF $>2,5$ & 4 & \multirow{2}{*}{ D } & RUIM \\
\hline $40 \leq \mathrm{IGGE} \leq 60$ e ICPF $\leq 2,5$ & 7 & \multirow{2}{*}{ PÉSSIMO }
\end{tabular}

Fonte: DNIT (2003).

Por fim, para a apresentação dos resultados, foi preenchido o Quadro de Resumos (Tabela 6), no qual demonstra a real situação em que o trecho escolhido apresenta, no que diz respeito às patologias. Com a análise deste quadro é possível identificar as condições de tráfego que a via se encontra. 
Após a avaliação de acordo com a norma DNIT 008/2003PRO, serão identificadas as possíveis origens da deterioração do pavimento no trecho analisado, juntamente com um levantamento de soluções técnicas para as patologias identificadas no trecho.

\section{RESULTADOS}

Foi executado o método do Levantamento Visual Contínuo na TO-255 trecho de Porto Nacional - TO a Monte do Carmo - TO, na qual utilizou como procedimento o que rege a norma DNIT 008/2003 - PRO, em que foram obedecidos os critérios de condições para realizar o mesmo.

O procedimento foi realizado no dia 01 de setembro de 2018 com início às 13 h40 e finalização às 17h40. Teve como equipe de operação o motorista e dois técnicos, sendo um deles o Engenheiro Civil Taffarel de Sousa Andrade CREA-TO no 209827/AP-TO. Os observadores procuraram relatar os pontos significantes e os tipos de patologias apresentadas ao longo da via.

Foi utilizado um carro equipado com velocímetro/odômetro calibrado a partir de um GPS, no qual obteve as coordenadas iniciais de N8817261, E785449 e coordenadas finais de N8808625, E815623. As condições climáticas eram favoráveis para o levantamento, uma vez que foi realizado em um dia ensolarado e com bastante luz natural.

O veículo estava manipulado a uma velocidade média aproximada de $40 \mathrm{~km} / \mathrm{h}$, percorrendo pela via em um único sentido, levando-se em consideração simultaneamente as duas faixas de tráfego. Foram analisados 40 subtrechos com extensão de $1 \mathrm{~km}$, uma vez que as condições do pavimento não se encontravam em perfeitas condições e para garantia de uniformidade da superfície examinada.

\section{Levantamento Visual Contínuo}

De acordo com o formulário do LVC, obtivemos o quantitativo de patologias encontradas no trecho. Através das tabelas que fornecem os valores dos pesos e frequências de defeitos, foi possível a realização do cálculo do Índice de Gravidade Global Expedido (IGGE), conforme Tabela 5.

Tabela 5: Formulário do IGGE.

\begin{tabular}{|c|c|c|c|c|c|c|c|c|c|c|c|c|c|}
\hline \multicolumn{4}{|c|}{ SEGMENTO } & \multicolumn{3}{|c|}{ TRINCAS } & \multicolumn{3}{|c|}{ DEFORMAÇÕES } & \multicolumn{3}{|c|}{$\begin{array}{l}\text { PANELA + } \\
\text { REMENDO } \\
\end{array}$} & \multirow{2}{*}{$\begin{array}{c}(\text { FtxPt })+(\text { FoapxPoap })+(\text { FprxPpr }) \\
=\text { IGGE }\end{array}$} \\
\hline $\begin{array}{l}\text { №. } \\
\text { SEG. }\end{array}$ & INÍCIO & FIM & EXT. & $\begin{array}{l}\mathrm{Ft} \\
\% \\
\end{array}$ & $\mathrm{Pt}$ & $\begin{array}{l}\text { Ft x } \\
\mathrm{Pt}\end{array}$ & $\begin{array}{c}\text { Foap } \\
\%\end{array}$ & Poap & $\begin{array}{c}\text { Foap x } \\
\text { Poap }\end{array}$ & $\begin{array}{l}\text { Fpr } \\
\text { no }\end{array}$ & Ppr & $\begin{array}{l}\text { Fpr x } \\
\text { Ppr }\end{array}$ & \\
\hline 1 & 0 & 1 & 1 & 0 & 0 & 0 & 0 & 0 & 0 & 2 & 0,7 & 1,4 & 1,4 \\
\hline 2 & 1 & 2 & 1 & 0 & 0 & 0 & 0 & 0 & 0 & 2 & 0,7 & 1,4 & 1,4 \\
\hline 3 & 2 & 3 & 1 & 0 & 0 & 0 & 0 & 0 & 0 & 5 & 0,8 & 4 & 4 \\
\hline 4 & 3 & 4 & 1 & 0 & 0 & 0 & 10 & 0,6 & 6 & 5 & 0,8 & 4 & 10 \\
\hline 5 & 4 & 5 & 1 & 0 & 0 & 0 & 10 & 0,6 & 6 & 4 & 0,8 & 3,2 & 9,2 \\
\hline 6 & 5 & 6 & 1 & 0 & 0 & 0 & 50 & 0,7 & 35 & 22 & 1 & 22 & 57 \\
\hline 7 & 6 & 7 & 1 & 0 & 0 & 0 & 0 & 0 & 0 & 20 & 1 & 20 & 20 \\
\hline 8 & 7 & 8 & 1 & 0 & 0 & 0 & 0 & 0 & 0 & 15 & 1 & 15 & 15 \\
\hline 9 & 8 & 9 & 1 & 0 & 0 & 0 & 0 & 0 & 0 & 10 & 1 & 10 & 10 \\
\hline 10 & 9 & 10 & 1 & 0 & 0 & 0 & 0 & 0 & 0 & 2 & 0,7 & 1,4 & 1,4 \\
\hline 11 & 10 & 11 & 1 & 0 & 0 & 0 & 0 & 0 & 0 & 30 & 1 & 30 & 30 \\
\hline 12 & 11 & 12 & 1 & 0 & 0 & 0 & 0 & 0 & 0 & 20 & 1 & 20 & 20 \\
\hline 13 & 12 & 13 & 1 & 0 & 0 & 0 & 0 & 0 & 0 & 5 & 0,8 & 4 & 4 \\
\hline 14 & 13 & 14 & 1 & 0 & 0 & 0 & 0 & 0 & 0 & 5 & 0,8 & 4 & 4 \\
\hline
\end{tabular}




\begin{tabular}{cccccccccccccc}
$\mathbf{1 5}$ & 14 & 15 & 1 & 0 & 0 & 0 & 0 & 0 & 0 & 5 & 0,8 & 4 & $\mathbf{4}$ \\
\hline $\mathbf{1 6}$ & 15 & 16 & 1 & 0 & 0 & 0 & 0 & 0 & 0 & 15 & 1 & 15 & $\mathbf{1 5}$ \\
\hline $\mathbf{1 7}$ & 16 & 17 & 1 & 0 & 0 & 0 & 10 & 0,6 & 6 & 20 & 1 & 20 & $\mathbf{2 6}$ \\
\hline $\mathbf{1 8}$ & 17 & 18 & 1 & 0 & 0 & 0 & 0 & 0 & 0 & 25 & 1 & 25 & $\mathbf{2 5}$ \\
\hline $\mathbf{1 9}$ & 18 & 19 & 1 & 0 & 0 & 0 & 0 & 0 & 0 & 15 & 1 & 15 & $\mathbf{1 5}$ \\
\hline $\mathbf{2 0}$ & 19 & 20 & 1 & 0 & 0 & 0 & 0 & 0 & 0 & 2 & 0,7 & 1,4 & $\mathbf{4}$ \\
\hline $\mathbf{2 1}$ & 20 & 21 & 1 & 0 & 0 & 0 & 0 & 0 & 0 & 5 & 0,8 & 4 & $\mathbf{4}$ \\
\hline $\mathbf{2 2}$ & 21 & 22 & 1 & 0 & 0 & 0 & 0 & 0 & 0 & 5 & 0,8 & 4 & $\mathbf{4}$ \\
\hline $\mathbf{2 3}$ & 22 & 23 & 1 & 0 & 0 & 0 & 0 & 0 & 0 & 5 & 0,8 & 4 & $\mathbf{1 , 4}$ \\
\hline $\mathbf{2 4}$ & 23 & 24 & 1 & 0 & 0 & 0 & 0 & 0 & 0 & 2 & 0,7 & 1,4 & $\mathbf{7 , 4}$ \\
\hline $\mathbf{2 5}$ & 24 & 25 & 1 & 0 & 0 & 0 & 10 & 0,6 & 6 & 2 & 0,7 & 1,4 & $\mathbf{5 3}$ \\
\hline $\mathbf{2 6}$ & $\mathbf{2 5}$ & 26 & 1 & 0 & 0 & 0 & 0 & 0 & 0 & 2 & 0,7 & 1,4 & $\mathbf{8 5}$ \\
\hline $\mathbf{2 7}$ & 26 & 27 & 1 & 0 & 0 & 0 & 40 & 0,7 & 28 & 25 & 1 & 25 & $\mathbf{6 6}$ \\
\hline $\mathbf{2 8}$ & 27 & 28 & 1 & 0 & 0 & 0 & 50 & 0,7 & 35 & 50 & 1 & 50 & $\mathbf{6 5}$ \\
\hline $\mathbf{2 9}$ & $\mathbf{2 8}$ & 29 & 1 & 0 & 0 & 0 & 10 & 0,6 & 6 & 60 & 1 & 60 & $\mathbf{4 0}$ \\
\hline $\mathbf{3 0}$ & $\mathbf{2 9}$ & 30 & 1 & 0 & 0 & 0 & 50 & 0,7 & 35 & 30 & 1 & 30 & $\mathbf{5 1}$ \\
\hline $\mathbf{3 1}$ & 30 & 31 & 1 & 0 & 0 & 0 & 0 & 0 & 0 & 40 & 1 & 40 & $\mathbf{3 0}$ \\
\hline $\mathbf{3 2}$ & 31 & 32 & 1 & 0 & 0 & 0 & 10 & 0,6 & 6 & 45 & 1 & 45 & $\mathbf{2 0}$ \\
\hline $\mathbf{3 3}$ & 32 & 33 & 1 & 0 & 0 & 0 & 10 & 0,6 & 6 & 22 & 1 & 22 & $\mathbf{3 , 2}$ \\
\hline $\mathbf{3 4}$ & 33 & 34 & 1 & 0 & 0 & 0 & 0 & 0 & 0 & 30 & 1 & 30 & $\mathbf{2 4}$ \\
\hline $\mathbf{3 5}$ & 34 & 35 & 1 & 0 & 0 & 0 & 0 & 0 & 0 & 20 & 1 & 20 & $\mathbf{3 5}$ \\
\hline $\mathbf{3 6}$ & 35 & 36 & 1 & 0 & 0 & 0 & 0 & 0 & 0 & 4 & 0,8 & 3,2 & $\mathbf{3 6}$ \\
\hline $\mathbf{3 7}$ & 36 & 37 & 1 & 0 & 0 & 0 & 0 & 0 & 0 & 24 & 1 & 24 & $\mathbf{0}$ \\
\hline $\mathbf{3 8}$ & 37 & 38 & 1 & 0 & 0 & 0 & 0 & 0 & 0 & 35 & 1 & 35 & \\
\hline $\mathbf{3 9}$ & 38 & 39 & 1 & 0 & 0 & 0 & 10 & 0,6 & 6 & 40 & 1 & 40 & 30 \\
\hline $\mathbf{4 0}$ & 39 & 40 & 1 & 0 & 0 & 0 & 0 & 0 & 0 & 30 & 1 & 30 & 0 \\
\hline $\mathbf{4 1}$ & 40 & 41 & 1 & 0 & 0 & 0 & 0 & 0 & 0 & 0 & 0 & 0 & \\
\hline
\end{tabular}

A última etapa do Levantamento Visual Contínuo é o quadro de resumos, no qual o Índice de Estado da Superfície do Pavimento (IES) indica um código e conceito sobre cada texto analisado de acordo com o Índice de Condição do Pavimento Flexível (ICPF) e do Índice de Gravidade Global Expedido (IGGE), como podemos visualizar os resultados na Tabela 6.

Tabela 6: Quadro de Resumos.

\section{SEGMENTO}

\section{Na. SEG. INÍCIO FIM EXT. ICPF IGGE}

RESULTADOS

VALOR CÓDIGO

BOM

REGULAR

REGULAR

REGULAR

REGULAR

RUIM

REGULAR

BOM

BOM

REGULAR

REGULAR

REGULAR

REGULAR

REGULAR

BOM

BOM

REGULAR

BOM

REGULAR

BOM

REGULAR

REGULAR

BOM

BOM

BOM
OBSERVAÇÃO

Sem Acostamento

Sem Acostamento

(20m Acostamento

Sem Acostamento
Entrada Chácara

Entrada Chácara

Sem Sinalização

Entrada Chácara

\begin{tabular}{llllllllll}
24 & 23 & 24 & 1 & 3 & 1,4 & 1 & B & BOM \\
\hline 25 & 24 & 25 & 1 & 3 & 7,4 & 1 & B & ВOM
\end{tabular}




\begin{tabular}{|c|c|c|c|c|c|c|c|c|c|}
\hline 26 & 25 & 26 & 1 & 4 & 1,4 & 0 & A & ÓTIMO & \\
\hline 27 & 26 & 27 & 1 & 2 & 53 & 5 & $\mathrm{D}$ & RUIM & Plantação de Soja \\
\hline 28 & 27 & 28 & 1 & 1 & 85 & 8 & $E$ & PÉSSIMO & Plantação de Soja \\
\hline 29 & 28 & 29 & 1 & 1 & 66 & 8 & $E$ & PÉSSIMO & Plantação de Soja \\
\hline 30 & 29 & 30 & 1 & 2 & 65 & 8 & $E$ & PÉSSIMO & \\
\hline 31 & 30 & 31 & 1 & 1 & 40 & 5 & $\mathrm{D}$ & RUIM & \\
\hline 32 & 31 & 32 & 1 & 1,5 & 51 & 5 & $\mathrm{D}$ & RUIM & \\
\hline 33 & 32 & 33 & 1 & 1,5 & 28 & 3 & $\mathrm{C}$ & REGULAR & \\
\hline 34 & 33 & 34 & 1 & 1,5 & 30 & 3 & $\mathrm{C}$ & REGULAR & \\
\hline 35 & 34 & 35 & 1 & 1,5 & 20 & 3 & $\mathrm{C}$ & REGULAR & \\
\hline 36 & 35 & 36 & 1 & 2 & 20 & 3 & $\mathrm{C}$ & REGULAR & \\
\hline 37 & 36 & 37 & 1 & 1 & 24 & 3 & $\mathrm{C}$ & REGULAR & \\
\hline 38 & 37 & 38 & 1 & 1 & 35 & 3 & $\mathrm{C}$ & REGULAR & \\
\hline 39 & 38 & 39 & 1 & 1 & 46 & 5 & $\mathrm{D}$ & RUIM & Pista Corrida de Cavalo \\
\hline 40 & 39 & 40 & 1 & 1 & 30 & 3 & $\mathrm{C}$ & REGULAR & Entrada Fazenda \\
\hline 41 & 40 & 41 & 1 & \multicolumn{5}{|c|}{ NÃO CONSIDERADO } & Cidade \\
\hline
\end{tabular}

Levando-se em consideração o LVC - método considerado - é possível concluir que o trecho analisado possui as seguintes características: dois por cento em estado Ótimo, situado no trecho 26, no qual o pavimento exibe apenas desgaste superficial, conforme Figura 2.

Vinte e cinco porcento em estado Bom, situado nos trechos 1, 8, 9, 15, 16, 18, 20, 23, 24, 25, na qual apresentam superficialmente patologias de remendo, desgaste, escorregamento, exsudação, escorregamento e deformações como afundamento e ondulações. Vale ressaltar que essas patologias se encontram aleatoriamente no decorrer do trecho e na maioria das vezes não são observadas respectivamente juntas.

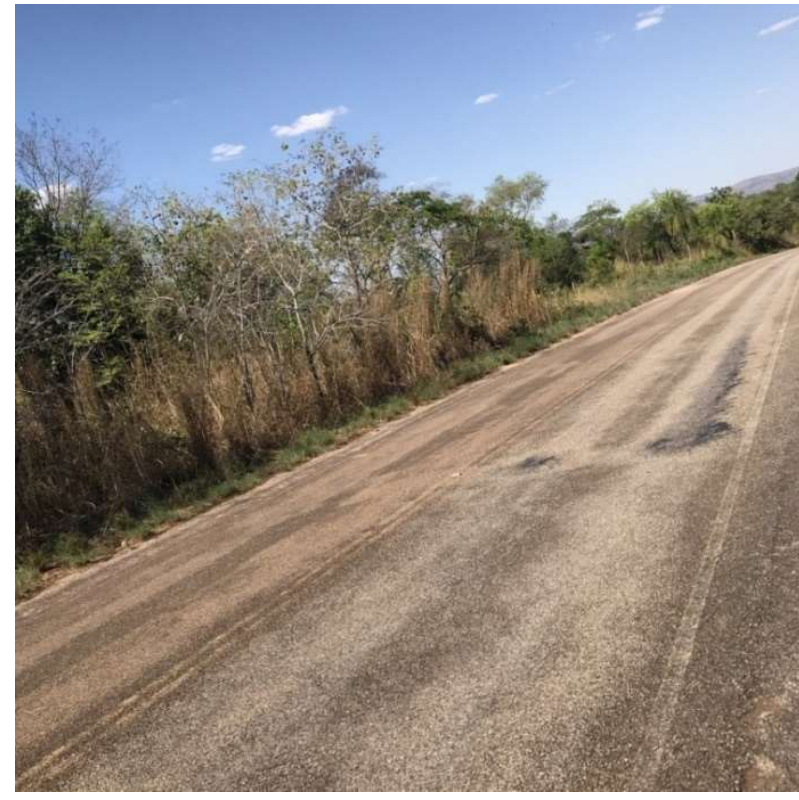

Figura 2: TO-255, Km 25, Trecho de Porto Nacional a Monte do Carmo (TO).

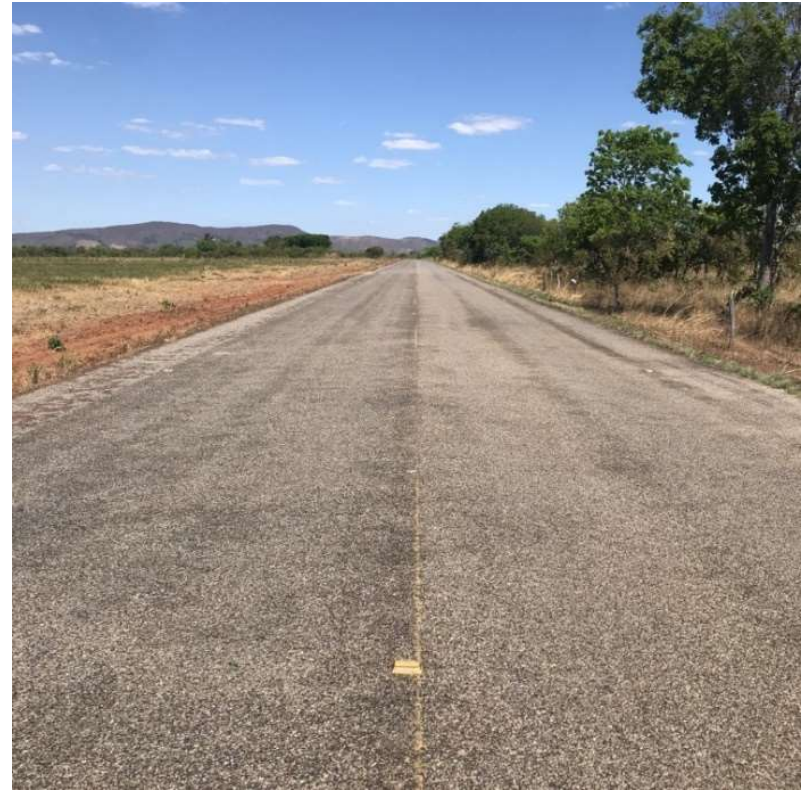

Figura 3: TO-255, Km 15, Trecho de Porto Nacional a Monte do Carmo (TO).

Cinquenta e dois porcento em estado Regular, localizado nos trechos $2,3,4,5,7,10,11,12,13,14$, $17,19,21,22,33,34,35,36,37,38,40$, onde o pavimento se encontra com irregularidade longitudinal ou transversal e com patologias pouco frequentes de remendo, desgaste, escorregamento, exsudação, escorregamento e deformações como afundamento e ondulações. 


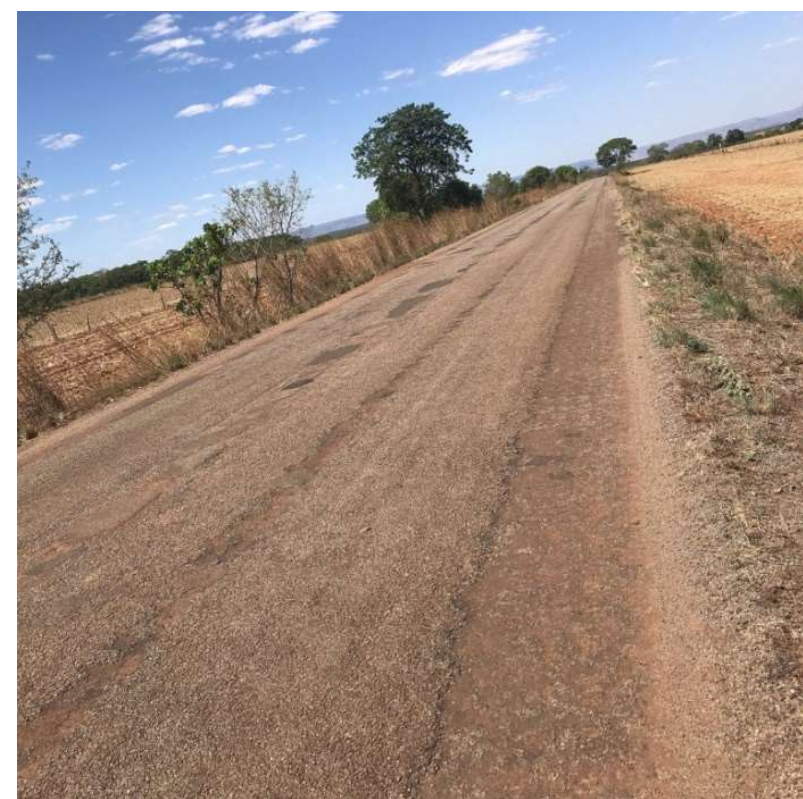

Figura 4: TO-255, Km 10, Trecho de Porto Nacional a Monte do Carmo (TO).

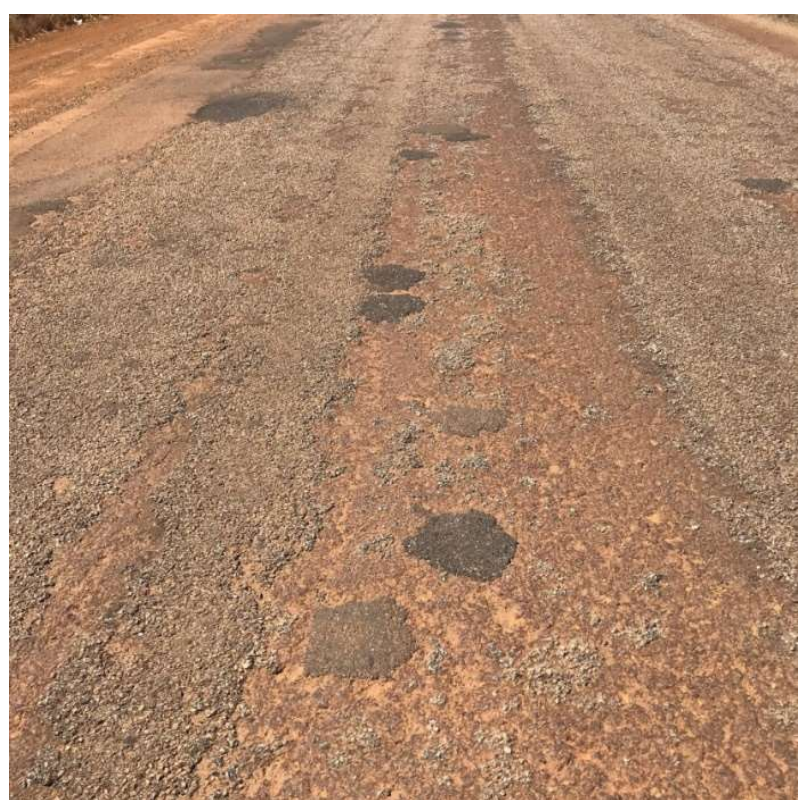

Figura 5: TO-255, Km 5, Trecho de Porto Nacional a Monte do Carmo (TO).

Treze porcento em estado Ruim, situado nos trechos 6, 27, 31, 32, 39, no qual o pavimento apresenta defeitos generalizados com correções prévias em áreas localizadas, sendo patologias superficiais ou profundas.

Oito porcento em estado Péssimo, localizado nos trechos 28,29 e 30, onde é notado defeitos generalizados com correções prévias em toda a extensão. Degradação do revestimento e das demais camadas - infiltração de água e descompactação da base. Através dos resultados encontrados no Quadro de Resumos (Tabela 6) foi possível produzir o gráfico da Figura 7.

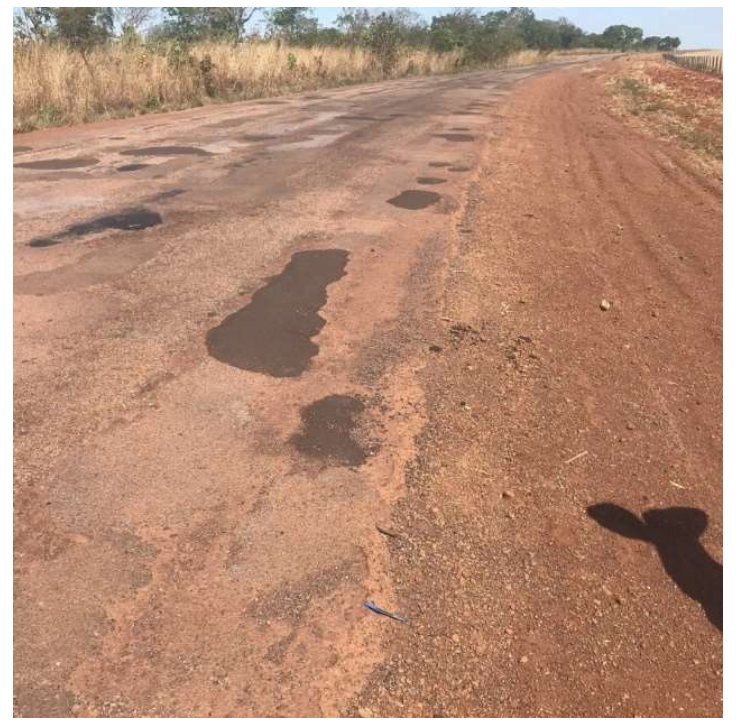

Figura 6 - TO-255, Km 27, Trecho de Porto Nacional a Monte do Carmo - TO.

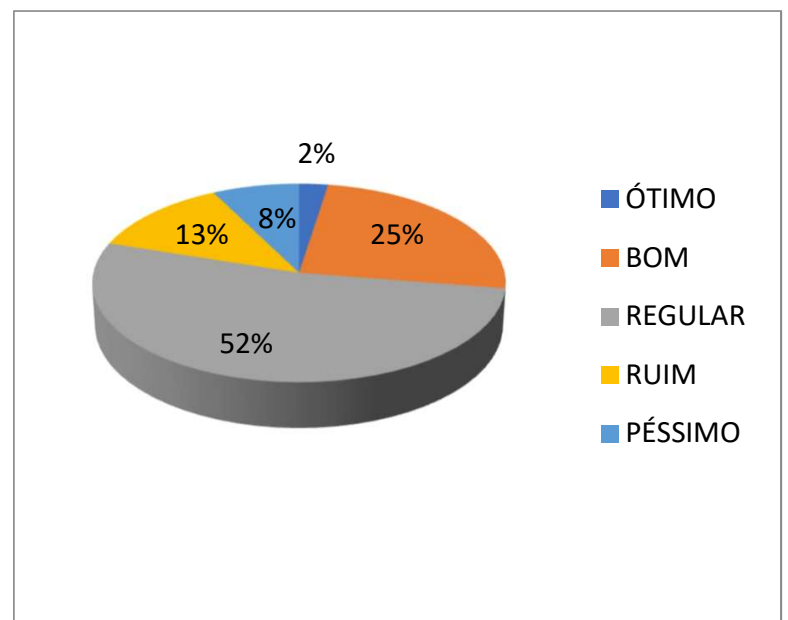

Figura 7: Gráfico de Qualidade dos Trechos.

\section{Manifestações Patológicas Encontradas}

A frequência de defeitos é registrada conforme a estimativa da qualidade e da porcentagem de ocorrência das patologias no trecho. Podemos observar na Tabela 7 que a frequência de defeitos considerada como Alta tem como principal propósito as patologias de remendo e desgaste. 
Tabela 7: Porcentagem de acordo com atuação de frequência de defeitos.

\begin{tabular}{|c|c|c|c|c|}
\hline & Sem Patologia & Baixa & Média & Alta \\
\hline Remendo & $0 \%$ & $15 \%$ & $5 \%$ & $80 \%$ \\
\hline Afundamento & $73 \%$ & $20 \%$ & $8 \%$ & $0 \%$ \\
\hline Ondulação & $85 \%$ & $13 \%$ & $3 \%$ & $0 \%$ \\
\hline Desgaste & $0 \%$ & $0 \%$ & $38 \%$ & $63 \%$ \\
\hline Exsudação & $10 \%$ & $48 \%$ & $35 \%$ & $8 \%$ \\
\hline Escorregamento & $13 \%$ & $40 \%$ & $23 \%$ & $25 \%$ \\
\hline
\end{tabular}

De acordo com os resultados do LVC, o Gráfico 1 a seguir, nos mostra de maneira mais clara os resultados da via analisada, sendo possível identificar o percentual de acordo com a frequência de defeitos (alta, média, baixa, sem patologia). A porcentagem está descrita de acordo com a atuação dos defeitos nos 40 subtrechos determinados.

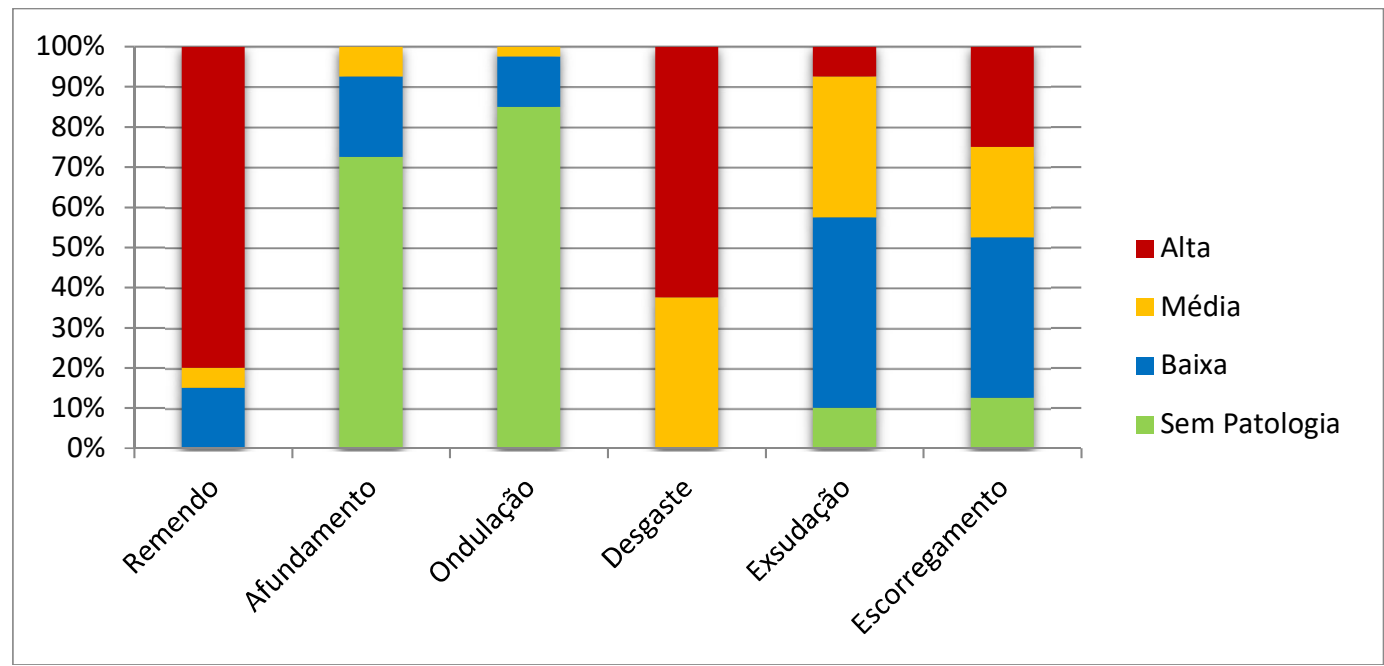

Figura 8: Frequência de defeitos por trecho.

Os defeitos de desgaste e remendo estão presentes em $100 \%$ dos trechos, sendo as manifestações patológica mais frequentes. Logo em seguida as exsudações com $90 \%$ de atuação. O escorregamento também aparece com porcentagem alta de 87,5\% de aparições nos trechos.

As manifestações patológicas menos frequentes foram os afundamentos com 27,5\% e as ondulações com 15\%. Deve-se levar em consideração que alguns dos subtrechos não possuíam acostamento da via, fator que o LVC coloca apenas em observação nas planilhas, mas não leva em consideração ao conceituar a condição do pavimento.

\section{DISCUSSÃO}

O trecho 26 que apresenta estado Ótimo de conservação, conforme o resultado do Levantamento Visual Contínuo, deve ser realizado uma conservação rotineira, na qual segundo o DNIT (2016) é um aglomerado de operações de conservação executadas com o fim de reparar ou evitar um defeito.

Nos trechos que apresentam estado Bom de conservação $(1,8,9,15,16,18,20,23,24,25)$, devem ser realizadas recuperações superficiais, na qual poderão ser utilizadas com o objetivo de corrigir pequenas falhas de trilha de roda. Método utilizado para selagem de trincas e restauração de aderência superficial 
(DNIT, 2006). Pode ser utilizada aplicações em lama asfáltica ou capa selante, com isso podendo evitar maiores problemas futuros e aumentando a vida útil do pavimento.

Nos trechos que apresentam estado Regular de conservação $(2,3,4,5,7,10,11,12,13,14,17,19$, $21,22,33,34,35,36,37,38,40$ ) devem ser realizados correções com pré-misturados à frio nas ondulações e afundamentos, lama asfáltica recapeamento. De acordo com o DNIT (2006), o recapeamento do pavimento baseia-se no revestimento realizado sobre outro já existente (compostas de betume e/ou concreto de cimento Portland), com o propósito de restaurá-lo ou reforçá-lo.

Os trechos em estudo que se apresentam em estado Ruim de conservação $(6,27,31,32,39)$ necessitam da realização de um recapeamento nesses segmentos, pois como foi classificado como sendo ruim, já possui inúmeras manifestações e não é viável ou recomendado recuperar isoladamente essas manifestações.

Os trechos em estudo que foram considerados como estado Péssimo de conservação $(28,29,30)$, necessitam de uma reconstrução total do pavimento, indo desde a camada de base e sub-base até a camada asfáltica. Por apresentaram defeitos generalizados com correções prévias por toda sua extensão.

Todos os trechos, ou seja, $100 \%$ dos pontos se encontram com manifestações patológicas de desgaste, o que evidencia a necessidade de uma implantação de medidas rotineiras e periódicas de forma adequada sobre a via. Caso seja realizado, o local jamais alcançará níveis de deterioração elevados nos quais sejam necessários a restauração ou reconstrução dela.

Sugere-se também a realização de sondagens nos trechos considerados críticos, a fim de realizar ensaios geotécnicos, compactação e CBR, com as coletas de amostras das camadas do pavimento. Realização do VMD (volume médio diário) da via, e restaurar os pontos de drenagens que se apresentam danificados.

\section{CONCLUSÃO}

Com base no Levantamento Visual Contínuo, as patologias com maior frequência nos trechos foram o Remendo, influenciados pela operação "tapa-buraco", e Desgaste com 100\% de presença nos trechos estudados. Seguidos pela exsudação com $90 \%$ de atuação e escorregamento com $87,5 \%$. Essas são as manifestações patológicas presentes em grande parte dos trechos.

As manifestações patológicas menos frequentes foram os afundamentos com $27,5 \%$ e as ondulações com $15 \%$. Conclui-se que a maioria dos trechos se caracteriza em estado Regular, no entanto, a presença de $100 \%$ de remendos e desgaste evidencia que o trecho precisa de um estudo a fundo, para que seja realizada a reparação de defeitos correta.

Realizando o método do LVC, pode-se perceber que nos cálculos do IGGE, índice este que é o fator preponderante para a classificação do estado do pavimento, a manifestação patológica de Desgaste não é levada em consideração nos cálculos, explicando a classificação regular do trecho. Se a patologia de desgaste fosse considerada pela norma, com certeza o estado do trecho estaria entre ruim e péssimo.

Por meio do Levantamento Visual Contínuo foi possível determinar as características da via divididas em subtrechos, com divisão de seguimos de $1 \mathrm{~km}$ cada. Os resultados obtidos foram que $2 \%$ do trecho se 
encontra em estado Ótimo, 25\% está em estado Bom, 52\% em estado Regular, 13\% em estado Ruim e $8 \%$ em estado Péssimo.

As alternativas de reparo vão desde uma pequena manutenção preventiva para correção de pequenas manifestações nos trechos em que se encontram em melhor condição, até uma reconstrução total para os piores trechos, a fim de aumentar a capacidade estrutural da superfície.

Concluindo esta pesquisa, é notória a importância de planejamentos que envolvam medidas de conservação e prevenção sobre o pavimento, evitando o agravamento das condições da via, possibilitando assim um gerenciamento consciente e eficaz.

\section{REFERÊNCIAS}

ALBANO JOÃO, F.. Efeitos dos excessos de carga sobre a durabilidade de pavimentos. Tese (Doutorado em Engenharia Civil) - Universidade Federal do Rio Grande do Sul, Porto Alegre, 2005.

BALBO JOSÉ T.. Pavimentação Asfáltica: materiais, projetos e restauração. São Paulo: Oficina de Textos, 2007.

BERNUCCI, L. B.; MOTTA, L.M.; CERATTI, J. A. P.; SOARES, J. B.. Pavimentação Asfáltica: Formação Básica para Engenheiros. Rio de Janeiro: 2006.

BRASIL. Lei n. 12.379 de 6 de janeiro de 2011. Dispõe sobre o Sistema Nacional de Viação - SNV - DNIT. Brasília: DOU, 2011.

BORGES, C. M.; SANTOS, M. J.; VIEIRA, E. T.. Tocantins: O crescimento e o Desenvolvimento Econômico Regional com a Criação do Novo Estado. Revista Gestão e Regionalidade, v.29, n.85, 2013.

CNT. Confederação Nacional do Transporte. 13 principais defeitos do pavimento das rodovias. 2018.

CNT. Confederação Nacional do Transporte. Relatório por unidade federativa. Edição 2017.

DNER. Departamento Nacional de Estradas de Rodagem. Conservação, restauração e melhoramentos - DNER - TER 02 - 79. Rio de Janeiro: 1979.

DNIT. Departamento Nacional de Infraestrutura de Transportes. Pavimentos Asfálticos: patologia e manutenção. São Paulo: Plêiade, 1997.

DNIT. Departamento Nacional de Infraestrutura de Transportes. Pavimentação - lama asfáltica - DNER ES 314/97. Rio de Janeiro: IPR, 1997.

DNIT. Departamento Nacional de Infraestrutura de Transportes. Defeitos nos pavimentos flexíveis e semirrígidos: terminologia. Norma DNIT 005/2003 - TER. Rio de Janeiro: 2003a.

DNIT. Departamento Nacional de Infraestrutura de Transportes. Levantamento visual contínuo para avaliação da superfície de pavimentos flexíveis e semirrígidos: procedimento. Norma DNIT 008 - PRO. Rio de Janeiro: 2003.

DNIT. Departamento Nacional de Infraestrutura de Transportes. Manual de Conservação Rodoviária. Rio de Janeiro: IPR, 2005.

DNIT. Departamento Nacional de Infraestrutura de Transportes. Pavimentos flexíveis - Recuperação de Defeitos em pavimentos: Especificação de serviço. Rio de Janeiro: 2009.

IBGE. Anuário Estatístico Do Brasil. Rio de Janeiro: IBGE, 2011

MELO, J. K. V.. Avaliação de serventia de um trecho da RN 118 a partir de levantamento visual contínuo. Monografia (Bacharelado em Engenharia Civil) - Universidade Federal Rural do Semiárido, Mossoró, 2014.

REIS, R. M. M.; TEIXEIRA, L. H.; CONSTANTINO, R., OMENA, W.. Manual Básico de Emulsões Asfálticas. 2 ed. Rio de Janeiro: ABEDA, 2010.

REIS, N. F. S.. Análise estrutural de pavimentos rodoviários: Aplicação a um pavimento reforçado com malha de aço. Dissertação (Mestrado em Engenharia Civil) - Universidade Técnica de Lisboa, Lisboa, 2009.

SILVA, P. F. A.. Manual de Patologia e Manutenção de Pavimentos. São Paulo: Pini, 2008.

SENÇO, W.. Manual de Técnicas de Pavimentação. 2 ed. São Paulo: Pini, 2007.

A CBPC - Companhia Brasileira de Produção Científica (CNPJ: 11.221.422/0001-03) detém os direitos materiais desta publicação. Os direitos referem-se à publicação do trabalho em qualquer parte do mundo, incluindo os direitos às renovações, expansões e disseminações da contribuição, bem como outros direitos subsidiários. Todos os trabalhos publicados eletronicamente poderão posteriormente ser publicados em coletâneas impressas sob coordenação da Sustenere Publishing, da Companhia Brasileira de Produção Científica e seus parceiros autorizados. Os (as) autores (as) preservam os direitos autorais, mas não têm permissão para a publicação da contribuição em outro meio, impresso ou digital, em português ou em tradução. 